\title{
Modeling the population dynamics and community impacts of Ambystoma tigrinum; a case study of phenotype plasticity
}

\author{
Maeve L. McCarthy ${ }^{\mathrm{a}, *}$, Dorothy Wallace ${ }^{\mathrm{b}}$, Howard H. Whiteman ${ }^{\mathrm{c}}$, Evan T. \\ Rheingold $^{\mathrm{b}}$, Ann M. Dunham ${ }^{\mathrm{b}}$, Olivia Prosper ${ }^{\mathrm{d}}$, Michelle Chen ${ }^{\mathrm{b}}$, Eileen Hu-Wang ${ }^{\mathrm{b}}$ \\ ${ }^{a}$ Department of Mathematics \& Statistics, Murray State University, Murray KY 42071 \\ ${ }^{b}$ Department of Mathematics, Dartmouth College, 27 N. Main Street, 6188 Kemeny Hall, \\ Hanover, NH 03755-3551 \\ ${ }^{c}$ Department of Biological Sciences, Murray State University, Murray KY 42071 \\ ${ }^{d}$ Department of Mathematics, 719 Patterson Office Tower, Lexington, KY 40506-0027
}

\begin{abstract}
Phenotypic plasticity is the ability of an organism to change its phenotype in response to changes in the environment. General mathematical descriptions of the phenomenon rely on an abstract measure of "viability" that, in this study, is instantiated in the case of the Tiger Salamander, Ambystoma tigrinum. This organism has a point in its development when, upon maturing, it may take two very different forms. One is a terrestrial salamander (metamorph)that visits ponds to reproduce and eat, while the other is an aquatic form (paedomorph) that remains in the pond to breed and which consumes a variety of prey including its own offspring.

A seven dimensional nonlinear system of ordinary differential equations is developed, incorporating small $(Z)$ and large $(B)$ invertebrates, Ambystoma young of the year $(Y)$, juveniles $(J)$, terrestrial metamorphs $(A)$ and aquatic paedomorphs $(P)$. One parameter in the model controls the proportion of juveniles maturing into $A$ versus

*Corresponding author

Email addresses: mmccarthy@murraystate.edu (Maeve L. McCarthy), dwallace@math.dartmouth. edu (Dorothy Wallace), hwhiteman@murraystate.edu (Howard H. Whiteman), Evan.T.Rheingold.17@dartmouth.edu (Evan T. Rheingold), Ann.M.Dunham.16@dartmouth.edu (Ann M. Dunham), olivia.prosper@uky.edu (Olivia Prosper), michen1115@gmail.com (Michelle Chen), eileen.huwang@alumni.dartmouth.edu (Eileen Hu-Wang)
\end{abstract}

Preprint submitted to Mathematical Biosciences

February 17, 2017

(C) 2017. This manuscript version is made available under the Elsevier user license http://www.elsevier.com/open-access/userlicense/1.0/ 
$P$. Solutions are shown to remain non-negative. Every effort was made to justify parameters biologically through studies reported in the literature.

A sensitivity analysis and equilibrium analysis of model parameters demonstrate that morphological choice is critical to the overall composition of the Ambystoma population. Various population viability measures were used to select optimal percentages of juveniles maturing into metamorphs, with optimal choices differing considerably depending on the viability measure. The model suggests that the criteria for viability for this organism vary, both from location to location and also in time. Thus, optimal responses change with spatiotemporal variation, which is consistent with other phenotypically plastic systems.

Two competing hypotheses for the conditions under which metamorphosis occurs are examined in light of the model and data from an Ambystoma tigrinum population at Mexican Cut, Colorado. The model clearly supports one of these over the other for this data set. There appears to be a mathematical basis to the general tenet of spatiotomporal variation being important for the maintenance of polyphenisms, and our results suggest that such variation may have cascading effects on population, community, and perhaps ecosystem dynamics because it drives the production of a keystone, cannibalistic predator.

Keywords: mathematical model, phenotype plasticity, Ambystoma tigrinum, predation, cannibalism, trophic ecology

\section{Highlights}

- Ambystoma tigrinum exhibits phenotypic plasticity with metamorph and paedomorph adults

- Nonlinear ODEs model food sources, young of the year, juveniles and both adult forms

- Morphological choice is critical to the overall composition of the Ambystoma population

- Population fitness measures indicate variability in optimal population distributions, consistent with polyphenic adaptation 


\section{Introduction}

Phenotypic plasticity is the ability of an organism to change its phenotype in response to changes in the environment. Although some regard phenotype plasticity as a mechanism of evolution [1], [2], it may also be viewed as subject to the laws of evolution like any other trait [3], [4]. Mathematical treatments relying on this perspective model evolutionary fitness in response to a varying selection gradient [5]. The resulting selection may be framed as an optimization problem for viability over varying selection pressure. Others argue that plasticity can slow or enhance genetic divergence [6] depending on the extent to which plasticity for a given organism is adaptive across the full range of its environments. Plasticity may in some circumstances exact a cost from the organism, which would also affect its viability [7], [8]. Clearly, whether plasticity is adaptive or not depends on the organism and the conditions of the various environments that host it.

Ambystoma tigrinum nebulosum, the Arizona tiger salamander, is a classic example of phenotypic plasticity. A species of salamander widespread in the U.S., the tiger salamander hatches from eggs into an aquatic larval form. In one growth trajectory, the larval form matures into an adult salamander capable of terrestrial life (metamorph), then becomes sexually mature. Another trajectory includes remaining in an aquatic form but maturing sexually to carry out the entire life cycle in the water (paedomorph) [9, 10]. Paedomorph adults are efficient foragers, and will prey on small members of their own species, as well as on invertebrates in their environment. Metamorphic adults may also occasionally eat young Ambystoma, but inefficiently and in low numbers $[11,12]$, and unpublished data.

Age-dependent models determine their categories based on age groups, while stagedependent models define their categories based on the life-cycle stage of the individuals [13]. This study uses a classification scheme, based on the characteristics of various stages of the life cycle of this species, to capture the most important ways in which individual members of a population change over time [14]. Additionally, the role of the individual in the food web of a pond changes during the life cycle. Vital rates such as birth, growth, maturation, fertility and mortality may change substantially, depending on the density of a particular prey or predator size class or on external factors such as the existence of predators. Such models should also thus include quantities describing other aspects of the food web, as interactions with these will affect the success of Ambystoma populations via intra- and intergenerational feedbacks.

Recent research has focused on the role of predation in affecting amphibian morpho- 
logical and behavioral plasticity, and such predator-prey interactions are considered a mediator of phenotypes. [15], [16], [17] [18]. These phenotypes may include traits that help an organism either avoid predators or assist in their own predation. How valuable such a trait will be depends on abundance of prey, abundance of predators, and other environmental features. Because of the complexity of the Ambystoma life cycle, the aquatic stages of this organism interact with all consumer trophic levels of a pond, sometimes as predator, sometimes prey, sometimes both [19, 20]. Structured population modeling is a useful tool to capture the range of population variation and its ecological and evolutionary implications over time. In particular, the tendency of paedomorphic adults to eat smaller members of their species should create an evolutionary incentive to produce a majority of metamorphs that leave the pond and seek food elsewhere. On the other hand, a permanent pond may be a less risky habitat for adults under some conditions, and may favor the production of paedomorphs. Understanding this potential trade-off, and its ecological and evolutionary implications, is the major thrust of this paper. To do so, we compare population viability values, defined as measures that allow us to evaluate population success and persistence.

This study addresses the following questions:

1. How important is the distribution of phenotypes to overall Ambystoma biomass, its population distribution among cohorts, and recruitment of young of the year?

2. How do different morphological dynamics compare under various measures of viability, and what tradeoffs are made at the population level to support plasticity?

3. How does the distribution of phenotypes affect the biomass of the trophic levels in the pond?

4. Does the model provide evidence that metamorphosis rates vary from year to year?

To this end a model is derived in Section 2 incorporating small $(Z)$ and large $(B)$ invertebrates, Ambystoma young of the year $(Y)$, juveniles $(J)$, adult metamorphs $(A)$ and paedomorphs $(P)$. One parameter in the model controls the proportion of juveniles maturing into $A$ versus $P$. Solutions are shown to remain non-negative.

The model constructed here is quite sensitive to the choice of parameters. Careless choice of parameters often drives the Ambystoma population to zero. Every effort 
was made to justify parameters biologically in Section 3.

The first two of these questions are studied through a sensitivity analysis and equilibrium analysis of model parameters. The second two questions are answered by varying the parameter associated with phenotype choice across its range. Section 4 describes the numerical experiments done with the model and reports the results. Section 5 discusses these results.

\section{Model formulation}

The model we will look at has several trophic levels. These levels are connected by birth, maturation, and predation relationships.

The young of the year (Y) mature into a sexually immature juvenile $(J)$. Juveniles $(J)$ can mature into paedomorphs $(P)$ which are sexually mature but aquatic, never developing legs and lungs, or else they $(J)$ can mature into terrestrial adults $(A)$ which can live on land or in water. Figure 1 describes maturation and reproduction of Ambystoma.

\section{Small $(Z)$ and large $(B)$ invertebrates.}

Minute organisms such as zooplankton $(Z)$ are at the bottom of the food chain. These are eaten by recently hatched salamander larvae, or "young of the year" $(Y)$, by juvenile salamanders $(J)$, and larger invertebrates $(B)$. Larger invertebrates $(B)$ are large enough to prey on smaller invertebrates, but too large to be eaten by young of the year Ambystoma larvae. Systemically, these function as competitors for $Z$, and an alternative food source for all stages of Ambystoma except young of the year $(J, P, A)$.

A fraction of small invertebrates $Z$ will mature into larger ones, represented as $B$. Both the growth rate of small invertebrates and the maturation rate of a fraction of these into larger invertebrates are controlled by a logistic term depending on their combined populations. This is a simplifying assumption that allows us to match the model to invertebrate biomass field data that does not distinguish between these sizes. Large invertebrates may die or exit the pond through maturation. This removal is modeled by a linear "death" rate which may be adjusted to give a representative ratio of large to small invertebrates. Invertebrate growth is assumed to occur through reproduction by $Z$ and an additional constant recruitment rate, $b$. This recruitment rate represents eggs laid by flying insects and also by $B$. The simplifying assumption that recruitment is constant reflects the assumption that most large invertebrates 


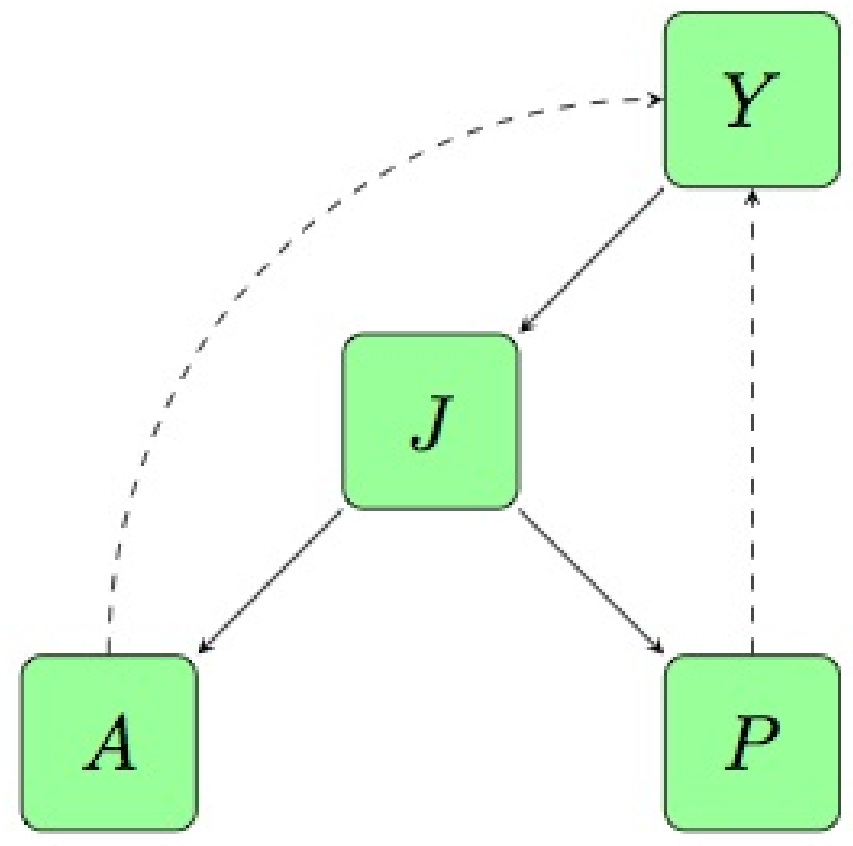

Figure 1: Salamander development flow chart. Solid arrows denote maturation from one stage to the next; dashed arrows denote contributions by the adult stages to the young-of-the-year $Y$ through reproduction.

will mature and exit the pond before returning to breed; thus direct birth by $B$ is ignored.

$Z^{\prime}=\operatorname{logistic}$ growth - maturation into $B$ - pred by $B$ - pred by $Y$ - pred by $J$

$B^{\prime}=\operatorname{logistic}$ growth \& maturation - predation by $P, A$ and $J$ - death

Ambystoma populations Young of the year $(Y)$ are eaten by paedomorphs $(P)$ and juveniles $(J)$. Juveniles $(J)$ are not discriminating eaters and will consume young of the year $(Y)$, as well as small and large invertebrates $(Z$ and $B)$. Metamorphs, or terrestrial adults $(A)$, develop lungs, are sexually mature, and may leave the pond to search for food or migrate. These are the creatures people think of as salamanders. They are large enough to eat large invertebrates $(B)$ but the model assumes they are too large to bother with small invertebrates $(Z)$. Metamorphic adults $(A)$ have 
been observed eating young of the year, but are reportedly inefficient at it; this effect was omitted from the model along with predation upon juveniles $(J)$. In addition the model includes an external source of food, $(e)$, because metamorphs spend most of their lives outside the pond ecosystem. Paedomorph adults $(P)$ derive all of their diet from within the pond. They are efficient predators eating large invertebrates, young of the year, and juveniles $(B, Y$, and $J)$. Table 1 summarizes the prey sources for each organism in the model.

\begin{tabular}{cr}
\hline Predator, $X$ & Food source, $P_{X}$ \\
\hline$Z$ & $\mathrm{n} / \mathrm{a}$ \\
$B$ & $\mathrm{Z}$ \\
$Y$ & $\mathrm{Z}$ \\
$J$ & $B+Y+Z$ \\
$P$ & $B+Y+J$ \\
$A$ & $B+e$
\end{tabular}

Table 1: Predator food sources

Ambystoma populations are controlled by the availability of prey, which affects both birth and maturation terms in the model. Note that due to the Ambystoma life stages, energy obtained by predation is returned to the system as maturation or birth, and thus does not necessarily add to the quantity of the consumer directly. Thus the energy flow does not represent strict mass action- for example the predation of $A$ on $B$ removes mass from $B$ but returns it to $Y$ through a birth rate proportional to the predation rate. Similarly, the predation of $J$ on $B$ removes mass from $B$ but returns it to the system in the form of maturation of $J$ to one of two adult forms. Figure 1 describes the trophic levels of the model with arrows indicating predation.

$Y^{\prime}=$ growth from $P \& A$ eggs - predation by $P, J$ - maturation to $J$ - death

$J^{\prime}=$ maturation from $Y$ - maturation to $P$ or $A$ - death

$A^{\prime}=$ maturation from $J$ - death

$P^{\prime}=$ maturation from $J$ - death

\section{Predation rates and functional responses}

Model assumptions about predation may be summarized as follows: 


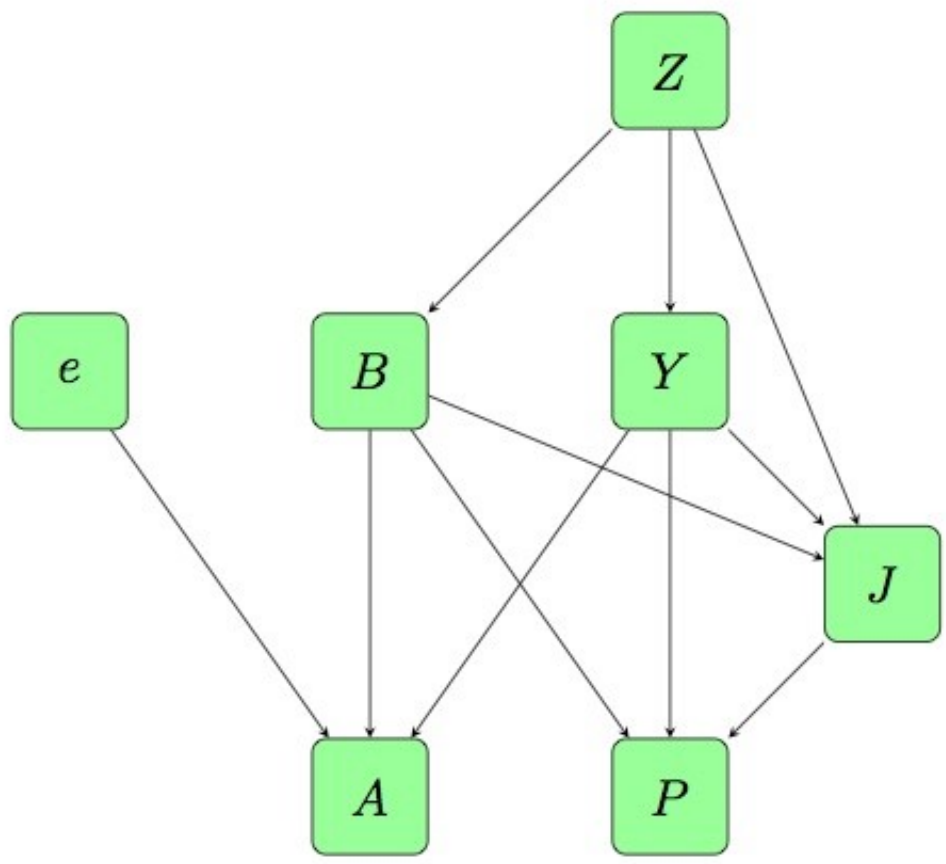

Figure 2: Trophic levels of the entire system, with the lowest trophic level at the top of the diagram. Arrows indicate predation. The quantity $e$ is a constant representing available food outside the pond.

1. Consumption rate of predator $X$ is capped by maximum daily rate $w_{X}$.

2. Predator $X$ has no preference and simply consumes prey in proportion to its representation in biomass.

3. Efficiency of consumption $h_{X}$ depends only on the predator, not the prey.

4. Predation follows by a type 2 functional response.

Except for zooplankton, the base of the food chain, all growth and maturation depends on food availability. As the normal size relations between trophic levels are generally stated in terms of biomass, this is the unit that will be employed in this model rather than individual organism counts. Specifying the model means finding maximum consumption, maturation, and reproductive rates for all organisms in the model in terms of wet biomass. Predation terms are the key to developing the model, 
which uses a type 2 functional response to give an expression for consumption per predator per day that approaches a maximum in the presence of abundant prey.

Suppose predator $\mathrm{X}$ eats a variety of prey, each denoted $U_{i}$, then the total biomass available to predator $\mathrm{X}$ is

$$
P_{X}=\Sigma_{i} U_{i}
$$

where $\Sigma_{i} U_{i}$ is the sum over all the prey of $X$ in units of biomass per unit volume.

We assume that the total predation of one unit of biomass of $\mathrm{X}$ on all of its possible prey is capped by the maximal consumption of prey biomass per unit biomass of predator $X$ per day, $\left(w_{X}\right)$ and thus represented by a modified Hill function:

$$
w_{X} H_{X}=w_{X} \frac{\Sigma_{i} U_{i}}{h_{X}+\Sigma_{i} U_{i}}=w_{X} \frac{P_{X}}{h_{X}+P_{X}}
$$

The constant $h_{X}$ is the half saturation value for this Hill function and represents the efficiency with which predator feeds on prey. Predators may be inefficient, efficient, or very efficient in this model, giving varying values of $h_{X}$ that depend on the predator, as has been observed in a general sort of way. It is also true that the same predator may be more efficient at catching some types of prey than others. As a simplifying assumption this model ignores that effect, although it would certainly be possible to incorporate it if the relevant biological measurements were available. Thus $h_{X}$ depends only on the predator, $X$.

The loss in prey $U_{i}$ biomass to predator $X$ is the product of the predation rate $\frac{w_{X} P_{X}}{h_{X}+P_{X}}=w_{X} H_{X}$ per predator, the fraction of all prey that is of type $U_{i}$, and the current predator $X$ biomass. Thus if $U_{i}$ is one of the quantities eaten by $X$, we have subtracted from the equation for $U_{i}^{\prime}$ the quantity

$$
w_{X} \frac{U_{i}}{\Sigma_{i} U_{i}} H_{X} X=w_{X} \frac{U_{i}}{h_{X}+\Sigma_{i} U_{i}} X
$$

Note that biomass of any given stage that is removed by predation is unavailable to mature. Hence predation has a systemic effect on maturation rates and need not be included explicitly in the next section.

Death, birth and maturation rates, and the choice of morphology 
We assume that mortality due to either natural causes or predation by predators external to the system is linear for each organism. This mortality is modeled as relative rates. The coefficients that determine those rates, e.g. $d_{X}$, may be taken as constants or time varying quantities, rather than density dependent quantities. Default constant rates are given.

The lowest trophic level, $Z$, grows as a logistic curve in the absence of predators with a recruitment term incorporated to account for larvae from sources external to the pond. The smaller insects, $Z$, are reduced by a relative maturation rate, $m_{Z B}$. The larger insects, $B$, are increased by a relative maturation rate, $m_{Z B}^{*}$. Because all organisms grow in size during their time in any given stage, the gain into compartment $B$ from maturation is set to be greater than the loss from compartment $Z$ by scaling by the ratio of average biomass of $B$ per average biomass of $Z$. Thus $m_{Z B}^{*}$ is just $m_{Z B}$ scaled by the appropriate factor.

For Ambystoma, if all of the $X$ mature into $W$ we get an input into the $W^{\prime}$ equation of the form

$$
m_{X W} H_{X} X
$$

where the constant $m_{X W}$ gives the maturation rate per unit time. This expression gives maximum rates of maturation when the organism is well fed, and no maturation in the absence of prey. Because all organisms grow in size during their time in any given stage, the gain into compartment $W$ from maturation is set to be greater than the loss from compartment $X$ by scaling by the ratio of average biomass of $W$ per average biomass of $X$. Thus $m_{X W}^{*}$ is just $m_{X W}$ scaled by the appropriate factor.

Only the Ambystoma quantities produce offspring in a different compartment from the parent. If $W$ gives birth to $Y$ we get an input to the $Y$ compartment of the form

$$
a_{Y W} H_{W} W
$$

labeling the constant $a_{Y W}$. Thus we have constants $a_{Y A}, a_{Y P}, a_{Y K}$.

The model includes one point of morphological choice. Juveniles have two options when maturing into adults: a terrestrial and an aquatic or paedomorphic option. The fraction becoming terrestrial adults is denoted $q_{J A}$. This may be taken as a con- 
stant or may be a function of the system or may be independent varying quantities, depending on the hypothesis under consideration.

\subsection{Full Equations}

Using this notation, the salamander population dynamics are modeled by the following system of ordinary differential equations:

$$
\begin{aligned}
& Z^{\prime}=\left(a_{Z} Z+b\right)\left(1-\frac{Z+B}{c_{Z+B}}\right)-m_{Z B} Z-\left(\frac{w_{Y} Y}{h_{Y}+P_{Y}}+\frac{w_{B} B}{h_{B}+P_{B}}+\frac{w_{J} J}{h_{J}+P_{J}}\right) Z \\
& B^{\prime}=m_{Z B}^{*} Z\left(1-\frac{Z+B}{c_{Z+B}}\right)-d_{B} B-\left(\frac{w_{J} J}{h_{J}+P_{J}}+\frac{w_{P} P}{h_{P}+P_{P}}+\frac{w_{A} A}{h_{A}+P_{A}}\right) B \\
& Y^{\prime}=a_{Y P} H_{P} P+a_{Y A} H_{A} A-d_{Y} Y-H_{Y} Y m_{Y}-\left(\frac{w_{J} J}{h_{J}+P_{J}}+\frac{w_{P} P}{h_{P}+P_{P}}\right) Y \\
& J^{\prime}=m_{Y J}^{*} H_{Y} Y-d_{J} J-H_{J} J\left[q_{J} m_{J A}+\left(1-q_{J}\right) m_{J P}\right]-\left(\frac{w_{P} P}{h_{P}+P_{P}}\right) J \\
& A^{\prime}=q_{J} m_{J A}^{*} H_{J} J-d_{A} A \\
& P^{\prime}=\left(1-q_{J}\right) m_{J P}^{*} H_{J} J-d_{P} P
\end{aligned}
$$

Default constants described in Section 3 and used in simulations are given in Table 1.

\subsection{Default parameters used in the model}

Carrying capacity Invertebrates in our study include both small organisms (Z) and larger ones (B). A carrying capacity is used for $Z+B$. A study by the EPA [21] includes invertebrate biomass measurements for invertebrates in a variety of ponds, including several from colder regions. Biomass is measured in two ways: by traps and sweep nets that catch floating organisms (maximum of $8524 \mathrm{mg} / \mathrm{m}^{3}$ in a lakeside prairie marsh in South Dakota), and by core samples that catch organisms on the bottom (maximum $5428 \mathrm{mg} / \mathrm{m}^{2}$, in a permanent pond). Combining these and assuming a pond of one meter in depth, we have a per $m^{3}$ carrying capacity for invertebrates in general of around 14 grams per $\mathrm{m}^{3}$, which is taken to be the default value of $c_{Z+B}$. 
Reproduction rate of $Z, a_{Z}$ As a proxy for small invertebrates, the model uses reported relative rates of brine shrimp increase in biomass per gram of biomass per day [22]. For the maximum growth rate for $Z$, we will use the maximum of these, setting $a_{Z}=.422$.

Maturation of $Z$ into $B, m_{Z B}$ and $m_{Z B}^{*} \mathrm{~A}$ fraction of small invertebrates will mature into larger ones, represented as $B$. The proxy organism, Telebasissa salva represents both $Z$ and $B$ when separated into two size classes, and has a maturation time of 31.4 days Runck et al [23]. Assuming half of small invertebrates will mature into large ones gives $m_{Z B}=.5 * 1 / 31.4=.016$. Because the organisms in the $B$ compartment are, on average, larger than those in $Z$, we must also scale the input to the $B$ compartment by the ratio of average biomass of $B$ to average biomass of $Z$. Johnston et al [24] gives body length and dry mass data on a range of invertebrates. The data for dry mass exhibit a bimodal distribution with a lower mode with dry mass $.188 \mathrm{mg}$ and higher mode with dry mass of .776. This ratio was used to give $m_{Z B}^{*}=(.776 / .188)\left(m_{Z B}\right)=.066$

Recruitment rate $b$ For relatively large values of $b$ the population drops rapidly to capacity, so the value of $b$ was set arbitrarily to .2 . reflecting abundant recruitment.

\section{Large invertebrate death rates not due to salamander predation, $d_{B}$.}

Wissinger et al [20] report differing ratios of large to small invertebrates in permanent versus autumnal basins, with permanent basins having higher peak densities of invertebrates, most of which are small. Autumnal basins have higher standing biomass of mostly large invertebrates. We took the reported ratio of standing biomass of autumnal to vernal ponds (1.86) as a proxy for the $B$ to $Z$ ratio at equilibrium for the ambystoma-free model. We then tuned the death rate $d_{B}$ to produce this ratio at equilibrium, giving a death rate of $d_{B}=0.002965$. Figure 3 shows the Ambystoma-free model run to equilibrium with these values.

Maximum consumption by Ambystoma per day, $w_{Y}, w_{J}, w_{A}, w_{P}, w_{B}$ Secor et al [25] give data on meal size as a percent of body mass for five adults of unspecified type. Each meal was consumed over a period of days, so we may assume the individuals were eating maximal amounts. The average daily consumption of these five individuals, as percent of their own body mass, is calculated at 1.307 percent. Thus we set all default values of the constants $w_{Y}, w_{J}, w_{C}, w_{A}, w_{P}, w_{K}$, equal to .013. This reflects an assumption that energy needs of the organism remain proportional to biomass throughout the life cycle. The maximum daily consumption for large invertebrates $\left(w_{B}\right)$ is harder to determine because $B$ represents an aggregate of relatively 


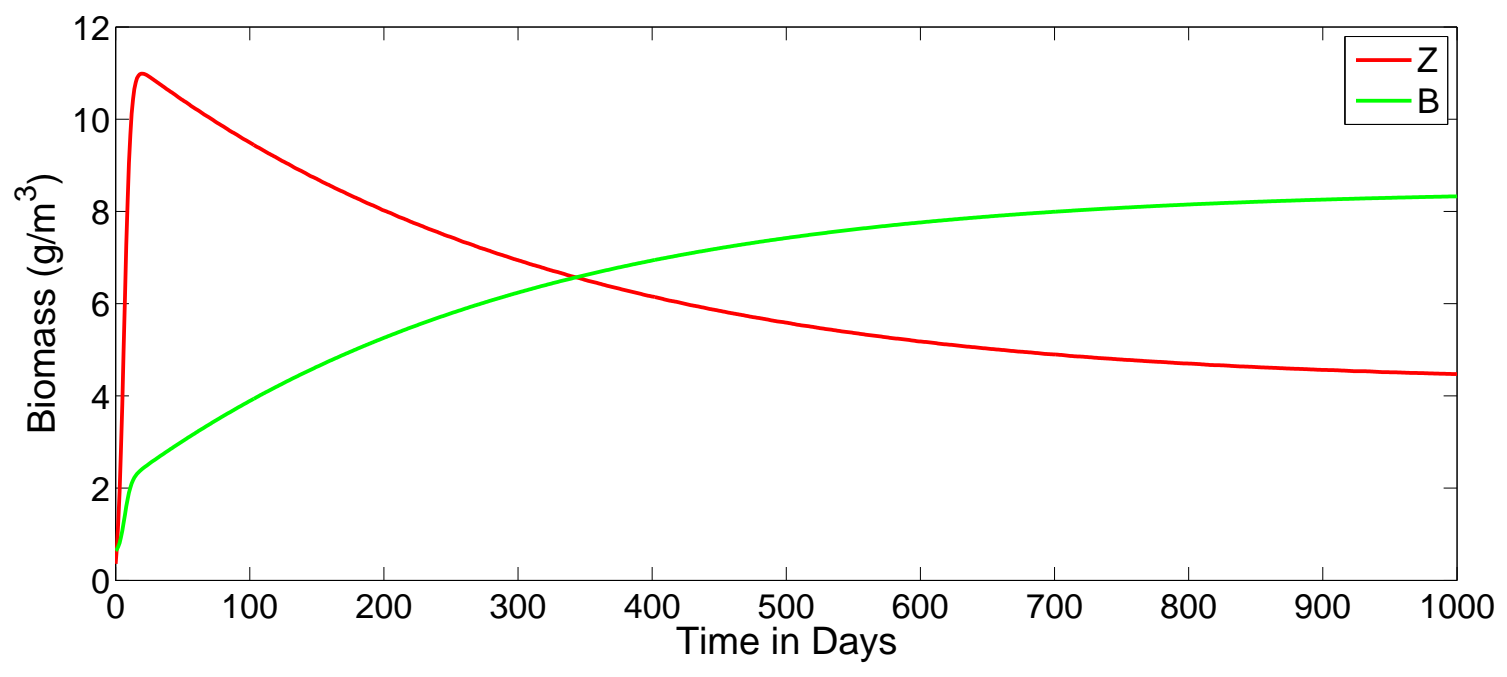

Figure 3: The model with no Ambystoma.

large invertebrate larvae. It is set to be equal to $w_{Y}$.

Half Saturation Rates, $h_{X}$ These control the efficiency of the functional response of predation to prey availability and arise in the Hill functions used to describe the predation rates (see equations (1)-(2)). Particularly efficient predators have a low value, which causes the predation rate to rise quickly in the presence of minimal prey. Typically these can only be measured in tank experiments in which the prey have few hiding places. However, relative efficiencies of organisms have been observed in the field. The values we assign for these parameters should be interpreted has having meaning only in their relationship to each other. As default, we set $h_{X}=.1$ for all Ambystoma, which represents an extremely efficient predator, for all predators except the less efficient $A$. where $h_{A}=1$. These choices reflect relative efficiencies, but not absolute efficiencies due to lack of data.

It is known that some organisms in the $B$ category consume young of the year salamanders, so $h_{B}$ is taken to be half of $h_{Y}$, or .005 .

External food sources for metamorphs, $e$ It is known that metamorphs $(A)$ has sources of food external to the pond. Data from Mexican Cut suggest that metamorphs consume an average of about $80 \%$ of their diet from terrestrial sources. A straightforward calculation shows that, at equilibrium, $e / B$ is the ratio of prey consumed by $A$ outside the pond to prey consumed by $A$ inside the pond. Equivalently, the fraction of prey from outside the pond is $e /(e+B)$. The model was run to 
equilibrium and the constant $e$ was tuned until the quantity $e /\left(e+B^{*}\right)=.8$. This gave $e=1.03$.

Explanation of constants: maturation rates of Ambystoma, $m_{Y J}, m_{J A}, m_{J P}$ and $m_{Y J}^{*}, m_{J A}^{*}, m_{J P}^{*}$

Maturation rates of this organism are known to vary greatly, from short seasonal maturation (120 days) in warm areas to 485 days or more in colder regions. Sexton and Bizer [26], [27] report larvae metamorphosing during the second warm season of their existence in montane Colorado, while Collins [28] reports larvae maturing at the end of a single growing season in Arizona. Furthermore, maturation rates must take into account the growth rates of the organism within a cohort, as all units in this study are in terms of biomass. A wide spread is reported for total time to maturity: 120 days to 485 days. As a default value, the model uses an intermediate value of 300 days. We will assume that $10 \%$ of this period is spent in the smallest stage $Y$, with the maturation period for $Y$ is 30 days, and the maturation period of $J$ is 270 days as default choices. This gives $m_{Y J}=.033$ per day and $m_{J A}=m_{J P}=.0037$ per day as default values. Wissinger et al [12] describe young of the year in the company of cannibalistic morphs in tank experiments. Wet biomass of these ranged from 1.25 to $3.75 \mathrm{~g}$. We will use a midrange biomass of $2.50 \mathrm{~g}$ as the default value.

Bizer [27] reports a range of sizes at metamorphosis (7.3 to $25.6 \mathrm{gm})$ for the cold habitat of Mexican Cut, Colorado. The majority of individuals were caught in the 11 to $13.5 \mathrm{gm}$ range. (Unpublished) data collected by Whiteman at Mexican Cut gives a range of 2 to 19.2 gms with a mean of 13.9 and median 14.1. We used the mean of 13.9 gms as average weight of a juvenile and as our representative biomass for juveniles $(J)$. The biomass increase in the $J$ compartment per unit biomass maturation of $Y$ is then approximately 13.9/2.5 $=5.56$ giving $m_{Y J}^{*}=.185$.

Secor [25] uses large adults weighing 30-34 gm in tank experiments. Unpublished data collected by Whiteman in Mexican Cut gives adult metamorph weights in a range of 3-56 gms with mean $25.6 \mathrm{gms}$, and paedomorph weights as 6.4-90 gms with mean 20.5 gms. We use a mean value of $25.6 \mathrm{gm}$ as our representative biomass for metamorphs, and mean value of $20.5 \mathrm{gms}$ for paedomorphs. The biomass increase in the $A$ compartment per unit biomass maturation of $J$ is then approximately $25.6 / 13.9=1.84$ giving $m_{J A}^{*}=.0068$. Similarly, $m_{J P}^{*}=.0055$.

Birth rates, $a_{Y A}, a_{Y P}$ Anderson et al [29] give total survivorship from egg deposition to metamorphosis as 3\%, indicating that egg mortality was high. Semlitsch [30] reports annual juvenile production from 0 to 30 individuals per breeding female per year. This corresponds to an average of 7.5 individuals per $22 \mathrm{gm}$ adult at $1.25 \mathrm{gm}$ 
per individual, per 365 days, for a relative birth rate of .0012 gms of larva biomass per gm adult biomass per day. $\left(a_{Y A}=a_{Y P}=.0012\right)$.

Density independent death rates, $d_{A}, d_{P}, d_{Y}, d_{J}$ Trenham [31] gives an overview of studies of annual survivorship of metamorphic adults (Ambystoma californiense), which ranges from $10 \%$ to more than $90 \%$ with a typical value of $60 \%$ per year. The $60 \%$ value gives a daily death rate of .0014 , calculated as $1-e^{\ln (.6) / 365}$. This is higher than the observed juvenile production rate given above, and therefore it is not biologically reasonable to include in the same model. A survival rate of $90 \%$ per year gives a daily mortality rate of .00029 . For the default value in this study we take $d_{A}=d_{P}=.00029$.

They note that their own observations indicated no relationship between adult size and survivorship, from which we infer that juveniles would experience similar mortality rates. However, our model explicitly includes intraspecies predation, which will make mortality higher in the juvenile categories. This seems likely to be a natural consequence as well as a biological one, so we are setting the same densityindependent mortality rate for juveniles, setting $d_{Y}=d_{J}=.00029$.

Morphological choice Upon maturation, normal juveniles $(J)$ may develop into metamorphs, which are terrestrial adults $(A)$, or else they may remain aquatic paedomorphs $(P)$. Again, hypotheses have been made about what controls this choice. However, for the purposes of setting a default quantity we will take $q_{J}=.5$ which will ensure that $50 \%$ of maturing $J$ become $A$ and $50 \%$ become $P$.

All default constants are summarized in Table 2.

\section{Methods and Results}

All simulations were conducted using Matlab software. With default parameters, a typical run is shown in Figure 4.

A sensitivity analysis was conducted by varying each parameter from its default level by $+/-10 \%$ and recording biomass values at equilibrium. This was done for three default choices of $q_{J}=.1, .5, .9$. Figures 5-7 show the resulting changes in equilibrium biomass of $A, P, J$, and $Y$ respectively, with respect to the most influential parameters $d_{Y}, h_{A}, h_{Y}$ and $q_{J}$. These simulations demonstrate the importance of the morphological choice parameter $q_{J}$ on the demographics of the equilibrium Ambystoma populations. Parameters not illustrated in Figures 5-7 had negligible impact on equilibrium values of any of the Ambystoma populations. 


\begin{tabular}{|c|c|c|}
\hline Parameter & Description & Default \\
\hline$c_{Z+B}$ & carrying capacity of $Z+B$ & $14 \mathrm{~g}$ wet biomass $/ \mathrm{m}^{3}$ \\
\hline$a_{Z}$ & relative birth rate of $\mathrm{Z}$ & $0.422 \mathrm{~g}$ per $\mathrm{g}$ per day, \\
\hline$b$ & recruitment rate of $Z$ & .20 \\
\hline$a_{Y A}$ & max. relative production rate of $\mathrm{Y}$ by $\mathrm{A}$ & $.0012 \mathrm{~g} / \mathrm{g}$ day \\
\hline$a_{Y P}$ & max. relative production rate of $\mathrm{Y}$ by $\mathrm{P}$ & .0012 \\
\hline$w_{B}$ & max. consumption by B per day & $.013 \mathrm{~g}=w_{Y}$ \\
\hline$w_{Y}$ & max. consumption by $\mathrm{Y}$ per day & $.013 \mathrm{~g}$ prey per $\mathrm{g} \mathrm{Y}$ wet biomass \\
\hline$w_{J}$ & max. consumption by J per day & $.013 \mathrm{~g}$ prey per $\mathrm{g} \mathrm{J}$ wet biomass \\
\hline$w_{A}$ & max. consumption by A per day & $.013 \mathrm{~g}$ prey per $\mathrm{g}$ A wet biomass \\
\hline$w_{P}$ & max. consumption by $\mathrm{P}$ per day & $.013 \mathrm{~g}$ prey per $\mathrm{g} \mathrm{P}$ wet biomass \\
\hline$h_{B}$ & half saturation for B per day & .005 (arb, extremely efficient case) \\
\hline$h_{Y}$ & half saturation for Y per day & .1 (arb, efficient case) \\
\hline$h_{J}$ & half saturation for $\mathrm{J}$ per day & .1 (arb, efficient case) \\
\hline$h_{A}$ & half saturation for A per day & 1 less efficient \\
\hline$h_{P}$ & half saturation for $\mathrm{P}$ per day & .1 (arb, efficient case) \\
\hline$m_{Z B}, m_{Z B}^{*}$, & max. maturation rate of $\mathrm{Z}$ into $\mathrm{B}$ & $.016, .0659$ \\
\hline$m_{Y J}, m_{Y J}^{*}$ & max. maturation rate of $\mathrm{Y}$ into $\mathrm{J}$ & $.033, .185$ \\
\hline$m_{J A}, m_{J A}^{*}$ & max. maturation rate of $\mathrm{J}$ into $\mathrm{A}$ & $.0037, .0068$ \\
\hline$m_{J P}, m_{J P}^{*}$ & max. maturation rate of $\mathrm{J}$ into $\mathrm{P}$ & $.0037, .0055$ \\
\hline$d_{B}$ & death rate of $\mathrm{B}$ including other predation & .002965 \\
\hline$d_{Y}$ & death rate of $Y$,including other predation & .00029 \\
\hline$d_{J}$ & death rate of $\mathrm{J}$ & .00029 \\
\hline$d_{A}$ & death rate of $\mathrm{P}$ & .00029 \\
\hline$d_{P}$ & death rate of $\mathrm{A}$ & .00029 \\
\hline$q_{J}$ & fraction of $\mathrm{J}$ maturing into form $\mathrm{A}$ & .5 \\
\hline$e$ & terrestrial food source for A & 1.03 \\
\hline
\end{tabular}

Table 2: Model parameters

The importance of $q_{J}$ to the overall behavior of the system was further explored by varying it across its full range and tracking Ambystoma equilibrium populations, both as a total and as percent of the whole. Equilibrium pattern diagrams in this parameter are shown for compartment biomasses at equilibrium (Figure 8), percent of total biomass at equilibrium (Figure 9) and biomass of the trophic levels at equilibrium 


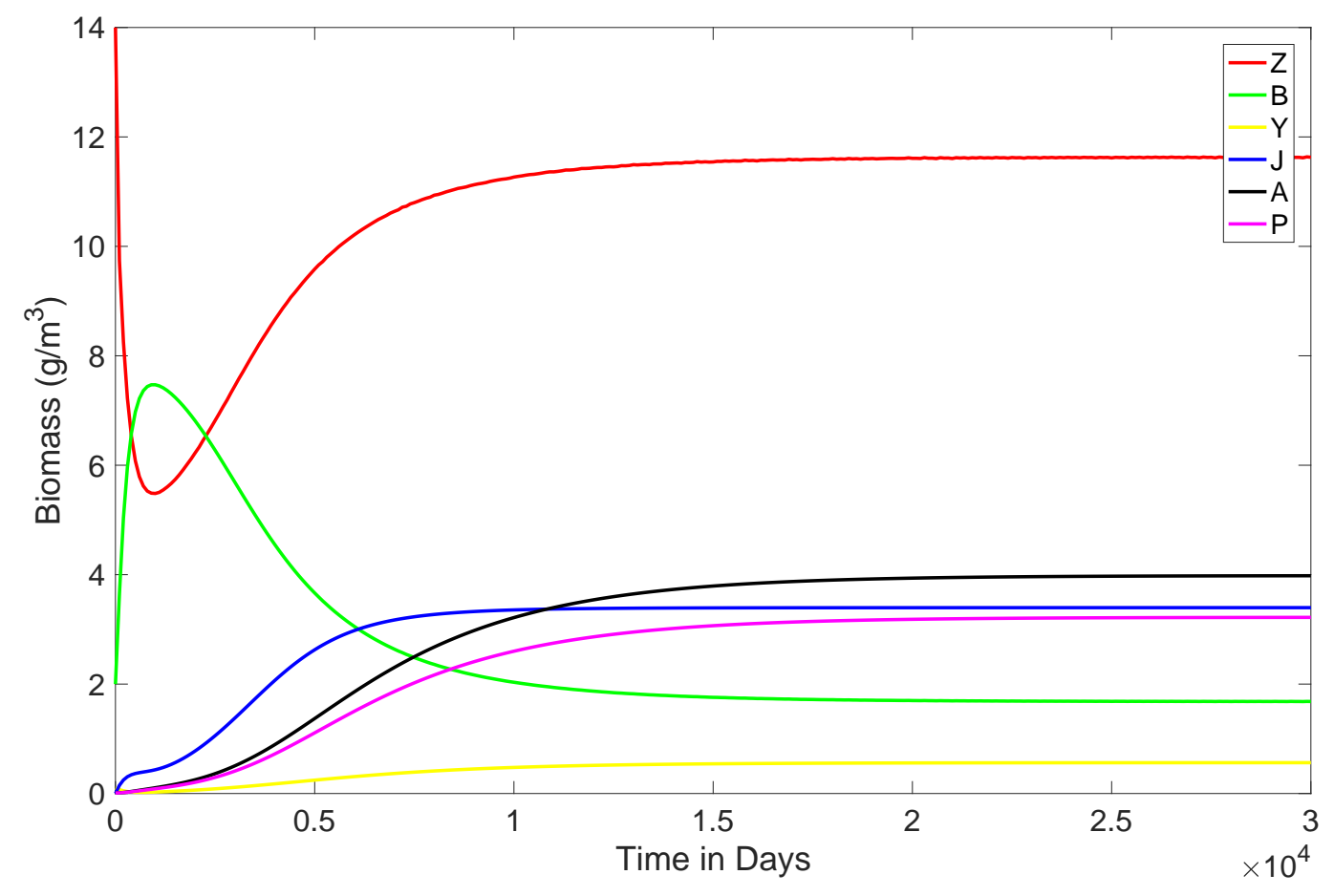

Figure 4: A typical run of the full model with default parameters and $A(0)=0.01, P(0)=$ $0.01, J(0)=0, Y(0)=0.1, B(0)=2, Z(0)=14$

(Figure 10).

\section{Discussion}

Returning to the questions raised in the introduction to this paper, it is now possible for the model to at least partially address them.

\subsection{Morphological choice is critical to the overall composition of the Ambystoma population.}

The per capita morphosis rate has a more significant influence on equilibrium ratios among phenotypes than other parameters, as illustrated in the diagrams of figures 5-7. Varying the morphological choice parameter, $q_{J}$, by plus or minus $10 \%$ from baseline runs at $q_{J}=.1, .5, .9$, had larger effect size than most other parameters. 

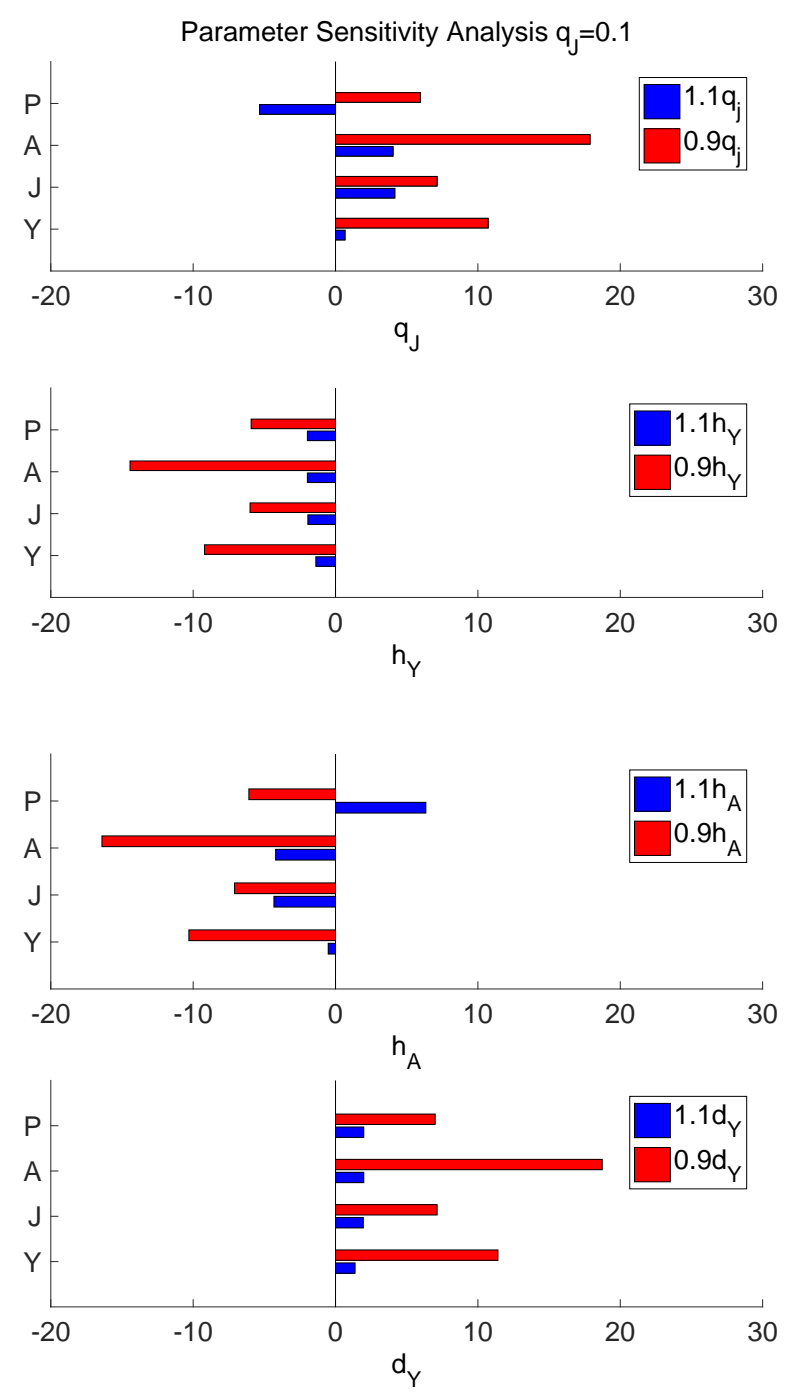

Figure 5: Sensitivity Analysis of $d_{Y}, h_{A}, h_{Y}$ and $q_{J}$ at $q_{J}=0.1$ with $q_{J}$ and $d_{A}$ demonstrating the most significant sensitivity. Blue bars indicate a parameter increase of $10 \%$. Red bars indicate a parameter decrease of $10 \%$.

Varying $q_{J}$ caused not only large changes in the distribution of adult phenotypes as expected (over $200 \%$ in figure 7 ), but also in the equilibrium biomass of young of the year, $Y$. Figure 7 shows the largest effect size at the highest metamorphic rate, and this trend is illustrated across a continuum of values in Figure 8.

Predation efficiency of $A$ and $Y$ are also important parameters. The parameters 
selected for this model, $h_{A}$ and $h_{Y}$, appear to be close to locally optimal. A slight increase in $h_{A}$ (less efficient predation) results in lower biomass for all Ambystoma population categories. A slight decrease in $h_{A}$ (more efficient predation) results in higher biomass for $A$ but lower biomass for all other categories. A slight increase or decrease in $h_{Y}$ results in lower biomass for all Ambystoma population categories. This is true at all three values of $q_{J}$ shown in Figures 5-7. Without specific measurements to justify this particular choice of parameter, it is still possible to note that this model suggests that optimal efficiencies of predation should exist. Metamorphs are assumed to be less efficient predators in the pond than paedomorphs [11]. The model suggests that a slight increase in predation efficiency for the metamorphic phenotype would have mostly negative consequences for the population as a whole.

\subsection{Morphological choice, various measures of viability, and viability tradeoffs at the population level}

It is possible to imagine various ways in which the paedomorph/metamorph distribution could vary in the relative costs and benefits to each morph [9]. The question of "What is natural selection trying to optimize" is likely to vary both between species and between locations within a species $[9,10]$. Several possibilities are discussed below.

Figure 8 shows that a system with no paedomorphs $\left(q_{J}=1\right)$ optimizes the total biomass of Ambystoma per cubic meter of aquatic habitat, and also optimizes young of the year. This is not surprising, as many salamanders do not have a paedomorphic form, likely because past selection has always favored metamorphosis. Because optimizing total biomass doesn't help explain the production of paedomorphs, it is probably not the best population viability measure for this organism.

A population that produces entirely metamorphs will have a larger total biomass than one producing paedomorphs, but such a population is more vulnerable to severe winters and terrestrial predators. If population viability depends on maximizing the chance of a pond being restocked the following year, it would favor a situation that maximizes the biomass of paedomorphs, as these cannot leave the pond and must reproduce locally. Figure 8 shows that this population is maximized at around $q_{J}=.9$. That is, $90 \%$ of juveniles are metamorphosing and only $10 \%$ remain in the pond as paedomorphs. Because paedomorphs consume young of the year, at low levels of $q_{J}$, where this form dominates, there is not as much recruitment to the next size level. For this model, a viability measure that favors the production of many 
paedomorphs actually favors a $90 \%$ rate of metamorphosis, and absolutely requires phenotype plasticity to do so.

In this model, resources are represented by $Z$ and $B$, the only compartments that are not part of the Ambystoma life cycle. Optimizing the use of these resources would result in a pond with Ambystoma being the largest possible percent of the biomass in that pond. Figure 9 shows an equilibrium pattern diagram for percent biomass. Again, a population producing entirely metamorphs maximizes the total percent of pond biomass given by Ambystoma populations, because that is where $Z$ and $B$ are minimized. Figure 9 shows that such a pond is mostly juveniles.

Perhaps a more useful distribution would be to maximize the resources devoted to adults in the pond, as these are most likely to restock that particular pond with young of the year. In this case, one would maximize the percent of biomass represented by the paedomorphic population, which occurs at approximately $q_{J}=0.45$, as in Figure 9. In this case the production of the two phenotypes is about equal.

Alternatively, it might be useful to maximize the resources devoted to recruitment of young of the year, $Y$. Figure 9 shows that the percent of biomass devoted to $Y$ is maximized at around $q_{J}=.85$. This is the point at which the consumption of young of the year by juveniles becomes a predominant feature of the pond, as the number of paedomorphs is relatively small.

At high rates of metamorphosis any improvement in the predation efficiency of metamorphs, $h_{A}$, becomes a significant and largely negative factor, as shown in Figure 7. The choices of $q_{J}$ that maximize various viability measures could change considerably, depending on the efficiency of metamorph predation within the pond. So it is possible that one measure of viability could be the ability to avoid predators. This would result in less predation by adults on young of the year, which would translate again into selection for low predation efficiency by adults. Metamorphosis is one way to achieve this, and leads to the possibility that the production of a high percentage of metamorphs is just a byproduct of selecting for the adult morph that is less likely to cannibalize their own offspring.

In this discussion the word "optimize" is used in its mathematical sense. We see that for certain choices of $q_{J}$, different subpopulations of ambystoma are "optimized", i.e. at their largest value. Whether these optima lead to increased viability of the overall population is not addressed by the model, although some possible scenarios are suggested. Similarly, the mechanisms by which a particular $q_{J}$ may be produced are left to a future paper. 


\subsection{Trophic levels and population dynamics.}

The biomass of trophic levels for a biological system are typically shown as a pyramid, with a relatively large biomass at the lowest trophic level, [32, 33]. The pyramid steps reduce in size moving up the trophic levels with the smallest amount of biomass devoted to top predators. By contrast, population dynamics at equilibrium generally have an inverted pyramid. Since less time is spent in the younger age categories, most of the biomass is devoted to adults, $[32,33]$. The organism modeled here is an interesting case, as the layers of the trophic pyramid coincide with the layers of the age structured pyramid. Considered by trophic levels, one would expect more biomass for young of the year, less for juveniles, and even less for adults. Considered as a population model, one would expect the reverse.

In this model the lowest trophic level is $Z$, followed by $Y+B$, then $J$, then $A+P$. Because $A$ also consumes prey from outside the pond, the top level is somewhat compromised. However the relationship among $Z, Y+B$, and $J$ is strictly within pond. Figure 10 shows how the relationship among these three varies with the morphological choice parameter, $q_{J}$.

At $q_{J}=.2$, where only $20 \%$ of juveniles metamorphose, the trophic levels are in the expected order, with $Z^{*}>B^{*}+Y^{*}>J^{*}$, while the population distribution is the reverse of usual. At $q_{J}=.8$, two of the trophic levels reverse, with $J^{*}>B^{*}+Y^{*}$. At this point all of the Ambystoma population biomasses are arranged in the expected order, with $A^{*}+P^{*}>J^{*}>Y^{*}$. At slightly higher levels of $q_{J}$ the juvenile population outstrips all of the lower trophic levels in biomass.

The model thus suggests a modification of the usual rules of thumb. When adult cannibals predominate, the equilibrium population distribution behaves like the usual expectation for trophic levels. When non-cannibal adults predominate, the population behaves more like the usual expectation for population models.

\subsection{Variation in morphological choice from year to year, and mechanisms for this variation}

The model discussed here treats the morphological choice parameter, $q_{J}$, as a constant, as a necessary first step in its analysis. However, it is known not to be constant, which is the source of the plasticity that allows it to vary as different viability measures come into play. As a rough measure of accuracy, equilibrium values of the model can be compared to unpublished data from Mexican Cut for annual censuses of a single permanent pond from 1990 to 2007, excluding those years without measurable 
numbers of young of the year, juveniles or paedomorphs. Ratios of populations can be compared to model predictions in those years when counts of all three cohorts were nonzero.

\begin{tabular}{crrr}
\hline Data sets & $\mathrm{A}$ & $B$ & $C$ \\
\hline average observed YOY/Juvenile & .2071 & .0894 & .0695 \\
comparable simulated $Y / J$ & .1339 & .092 & .0809 \\
average observed Juvenile/Paedomorph & 2.877 & .494 & 8.842 \\
comparable simulated $J / P$ & 3.5272 & .5874 & 10.6610 \\
best fit $q_{J}$ & .85 & .1 & .95 \\
Metamorphosis rate & $85 \%$ & $10 \%$ & $95 \%$ \\
Paedomorphosis rate & $15 \%$ & $90 \%$ & $5 \%$ \\
\hline
\end{tabular}

Table 3: Comparison of field data to model outputs via cohort ratios $Y / J$ and $J / P$. A) All years in which three cohorts were present, B) Excluding additional years with very large populations of young of the year or juveniles, C) Two outlier years with unusually large numbers of juveniles.

Including all years with three cohorts present, the data from Mexican Cut is best approximated at $q_{J}=.85$. This $85 \%$ rate of metamorphosis is exactly in line with a strategy that optimizes paedomorphs in the model.

Excluding additional outlier years in which either juvenile or young of the year populations were very large, the data is approximated by the model at $q_{J}=.1$. A $10 \%$ rate of metamorphosis is not optimal by any of the viability measures discussed above, and suggests a dynamic that is not accounted for by simple natural selection arguments.

It is likely that $q_{J}$ is varying with population and ecosystem dynamics. This hypothesis is supported by the fact that, at the two outlier years for $J$, the observed cohort ratios are approximated by the model at $q_{J}=.95$, a high rate of metamorphosis (or correspondingly low rate of paedomorphosis).

There are several hypotheses used to explain the life-stage choice of paedomorphosis versus metamorphosis. In particular, the "paedomorphic advantage" mechanism assumes that if aquatic growing conditions are good then paedomorphosis is selected. Large strong larvae become paedomorphs to take advantage of the aquatic environment. Small weak larvae become metamorphs to escape competition with larger individuals within the pond. For this hypothesis, the per capita metamorphosis rate is positively density dependent (increasing) [9], [34]. Alternatively, the "best of a 
bad lot" mechanism assumes that paedomorphosis occurs when growing conditions are poor and the larvae do not become large enough to metamorphose [34]. For this hypothesis, the per capita metamorphosis rate is negatively density dependent (decreasing) [34]. The model developed here suggests that in years of high population density there is a higher rate of metamorphosis (meaning a lower rate of paedomorphosis), supporting the "paedomorphic advantage" hypothesis, and giving evidence against the "best of a bad lot" mechanism.

The introduction of functional dependence of the morphological choice parameter $q_{J}$ on $J, A$ or $P$ would give a better explanation of the data from Mexican Cut, and a model taking into account years of zero recruitment might give a different picture of optimal population strategies. In particular, by comparing model output to data sets it may be possible to distinguish whether $q_{J}$ is a function of population density, as argued by the two hypotheses described above, or more directly a function of prey density.

\subsection{Directions for further research}

The model and results presented here are necessarily the precursor to further research. We describe some future directions in this section, although it is easy to imagine many more.

As with any model, decisions were made to exclude details for which supporting biological detail was not found. Grouping insects into just two compartments is one such simplification, and assuming constant maturation and death rates is another. Extensions of this model could be used to address the implications of more refined insect population dynamics and the role of temperature in regulating maturation and death rates.

Modeling the effect of temperature and habitat availability, in particular, would allow comparisons of Ambystoma populations in different regions of the country. The model could conceivably explain some of the macroscopic differences observed in population dynamics among different ecosystems such as the relatively cold and permanent habitat at Mexican Cut versus the warmer, transient habitats of the Texas playas.

The parameter $(q)$ that governs morphological choice was varied during this study, but a more interesting and accurate way to handle morphological determination would be to make this parameter a function of the environment (e.g. food supply

or crowdedness or temperature). Extending the model in this way would allow one 
to test various hypotheses about the trigger for morphological choice by comparing model predictions with field observation.

Many Ambystoma populations suffer shocks to the system, such as when a flock of birds settles on a playa in West Texas. The sudden appearance and disappearance of large numbers of predators will have a big impact on the population of a given playa, and the impact is likely to differ depending on seasonality. The model developed here, with suitable extensions, lends itself to studying the resilience of Ambystoma populations to sudden population drops.

\section{Conclusion}

In sum, our modeling efforts have allowed us to explore the population, community and evolutionary implications of a polyphenic, predatory salamander. Morph production and thus frequency in the population had a large impact on both population and community metrics. Our results have also shown the viability payoffs to each morph may vary in both space and time, which is consistent with empirical studies of polyphenism, $[9,35,10]$. Thus, there appears to be a mathematical basis to the general tenet of spatiotomporal variation being important for the maintenance of polyphenisms, producing different optimal solutions in different environments. Our results suggest that such variation may have cascading effects on population, community, and perhaps ecosystem dynamics because it drives the production of a keystone, cannibalistic predator. Because of the critical importance of understanding such cascading affects in biological systems, [36], further analyses of this and similar systems are warranted.

Acknowledgements. This research was funded by the Murray State University Committee on Institutional Studies and Research (CISR), two CISR Presidential Research Fellowships, several RMBL Fellowships, and the National Science Foundation (DEB0109436 and DEB-1354787 to HW; EPI-0132295 to G. Kipphut; and UBM-0531865 and UBM-1028125 to R. Fister), and by the Dartmouth College Student Internship program.

[1] C. Schlichting, The role of phenotypic plasticity in diversification, Phenotypic Plasticity: functional and conceptual approaches (2004) 191-200.

[2] M. Pigliucci, C. J. Murren, Perspective: Genetic assimilation and a possible evolutionary paradox: can macroevolution sometimes be so fast as to pass us by?, Evolution 57 (7) (2003) 1455-1464. 
[3] J. A. Endler, Natural selection in the wild, no. 21, Princeton University Press, 1986.

[4] S. Via, Adaptive phenotypic plasticity: target or by-product of selection in a variable environment?, American Naturalist (1993) 352-365.

[5] G. De Jong, Evolution of phenotypic plasticity: patterns of plasticity and the emergence of ecotypes, New Phytologist 166 (1) (2005) 101-118.

[6] B. M. Fitzpatrick, Underappreciated consequences of phenotypic plasticity for ecological speciation, International Journal of Ecology 2012.

[7] X. THIBERT-PLANTE, A. Hendry, The consequences of phenotypic plasticity for ecological speciation, Journal of evolutionary biology 24 (2) (2011) 326-342.

[8] T. J. DeWitt, A. Sih, D. S. Wilson, Costs and limits of phenotypic plasticity, Trends in Ecology \& Evolution 13 (2) (1998) 77-81.

[9] H. H. Whiteman, S. A. Wissinger, A. J. Bohonak, Seasonal movement patterns in a subalpine population of the tiger salamander, ambystoma tigrinum nebulosum, Canadian Journal of Zoology 72 (10) (1994) 1780-1787.

[10] M. Denoël, P. Joly, H. H. Whiteman, Evolutionary ecology of facultative paedomorphosis in newts and salamanders, Biological Reviews 80 (2005) 1-9.

[11] H. H. Whiteman, S. A. Wissinger, W. S. Brown, Growth and foraging consequences of facultative paedomorphosis in the tiger salamander, ambystoma tigrinum nebulosum, Evolutionary Ecology 10 (4) (1996) 433-446.

[12] S. A. Wissinger, H. H. Whiteman, M. Denoël, M. L. Mumford, C. B. Aubee, Consumptive and nonconsumptive effects of cannibalism in fluctuating agestructured populations, Ecology 91 (2) (2010) 549-559.

[13] H. Caswell, Matrix Population Models: Construction, Analysis, and Interpretation, Sinauer Associates, Sunderland, Massachusetts, 2001.

[14] S. N. Wood, Inverse problems and structured-population dynamics., in: S. Tuljapurkar, H. Caswell (Eds.), Structured-Population Models in Marine, Terrestrial and Freshwater Systems., Chapman and Hall, 1997, pp. 555-586.

[15] R. A. Relyea, J. R. Auld, Predator-and competitor-induced plasticity: how changes in foraging morphology affect phenotypic trade-offs, Ecology 86 (7) (2005) 1723-1729. 
[16] W. C. Kerfoot, A. Sih, Predation: direct and indirect impacts on aquatic communities, University Press of New England, 1987.

[17] R. Tollrian, C. D. Harvell, The ecology and evolution of inducible defenses, Princeton University Press, 1999.

[18] P. Barbosa, I. Castellanos, Ecology of predator-prey interactions, Oxford University Press, 2005.

[19] J. R. Holomuzki, J. P. Collins, P. E. Brunkow., Trophic control of fishless ponds by tiger salamander larvae, Oikos 71 (1994) 55-64.

[20] S. A. Wissinger, A. J. Bohonak, H. H. Whiteman, W. S. Brown, Subalpine wetlands in Colorado: Habitat permanence, salamander predation and invertebrate communities, in: D. P. Bazter, S. A. Wissinger (Eds.), Invertebrates in Freshwater Wetlands of North America: Ecology and Management, John Wiley and Sons, 1999.

[21] U. S. E. P. Agency", Bioindicators for assessing ecological integrity of prairie wetlands, Report EPA/ 600/ R-96/ 082 (September 1995).

[22] J. Dhont, P. Lavens, Tank production and use of ingrown artemia, Food and Agriculture Organization of the United Nations, Fisheries and Aquaculture Department.

[23] C. Runck, W. Blinn, Secondary production by telebasis salva (odonata) in a thermally constant aquatic ecosystem, Journal of the North American Benthological Society (1993) 136-147.

[24] T. A. Johnston, R. A. Cunjak, Dry mass-length relationships for benthic insects: a review with new data from catamaran brook, new brunswick, canada, Freshwater Biology 41 (4) (1999) 653-674.

[25] S. M. Secor, M. Boehm, Specific dynamic action of ambystomatid salamanders and the effects of meal size, meal type, and body temperature, Physiological and Biochemical Zoology 79 (4) (2006) 720-735.

[26] O. J. Sexton, J. R. Bizer, Life history patterns of ambystoma tigrinum in montane colorado, American Midland Naturalist (1978) 101-118.

[27] J. R. Bizer, Growth rates and size at metamorphosis of high elevation populations of ambystoma tigrinum, Oecologia 34 (2) (1978) 175-184. 
[28] J. P. Collins, Distribution, habitats and life history variation in the tiger salamander, ambystoma tigrinum, in east-central and southeast arizona, Copeia (1981) 666-675.

[29] J. D. Anderson, D. D. Hassinger, G. H. Dalrymple, Natural mortality of eggs and larvae of ambystoma t. tigrinum, Ecology (1971) 1107-1112.

[30] R. Semlitsch, D. Scott, J. Pechmann, J. Gibbons, Structure and dynamics of an amphibian community: evidence from a 16-year study of a natural pond, Longterm studies of vertebrate communities. Academic Press, San Diego, California (1996) 217-248.

[31] P. C. Trenham, H. Bradley Shaffer, W. D. Koenig, M. R. Stromberg, S. Ross, Life history and demographic variation in the california tiger salamander (ambystoma californiense), Copeia 2000 (2) (2000) 365-377.

[32] R. Ricklefs, R. Relyea, Ecology: The Economy of Nature, 7th Edition, W. H. Freeman, 2013.

[33] M. Begon, R. W. Howarth, C. R. Townsend, Essentials of Ecology, 4th Edition, Wiley, 2014.

[34] H. Whiteman, S. A. Wissinger, M. Denoël, C. Mecklin, N. Gerlanc, J. Gutrich, Larval growth in polyphenic salamanders: making the best of a bad lot, Oecologia 168 (1) (2012) 109-118.

[35] D. A. Roff, The evolution of threshold traits in animals, The Quarterly Review of Biology 71 (1996) 3-35.

[36] J. Terborgh, J. A. Estes, Trophic cascades: Predators, prey, and the changing dynamics of nature, Island Press, Washington, D.C., 2010.

[37] K. L. Larson, W. Duffy, E. Johnson, M. F. Donovan, M. J. Lannoo, paedocannibal morph barred tiger salamanders (ambystoma tigrinum mavortium) from eastern south dakota, The American midland naturalist 141 (1) (1999) 124-139. 

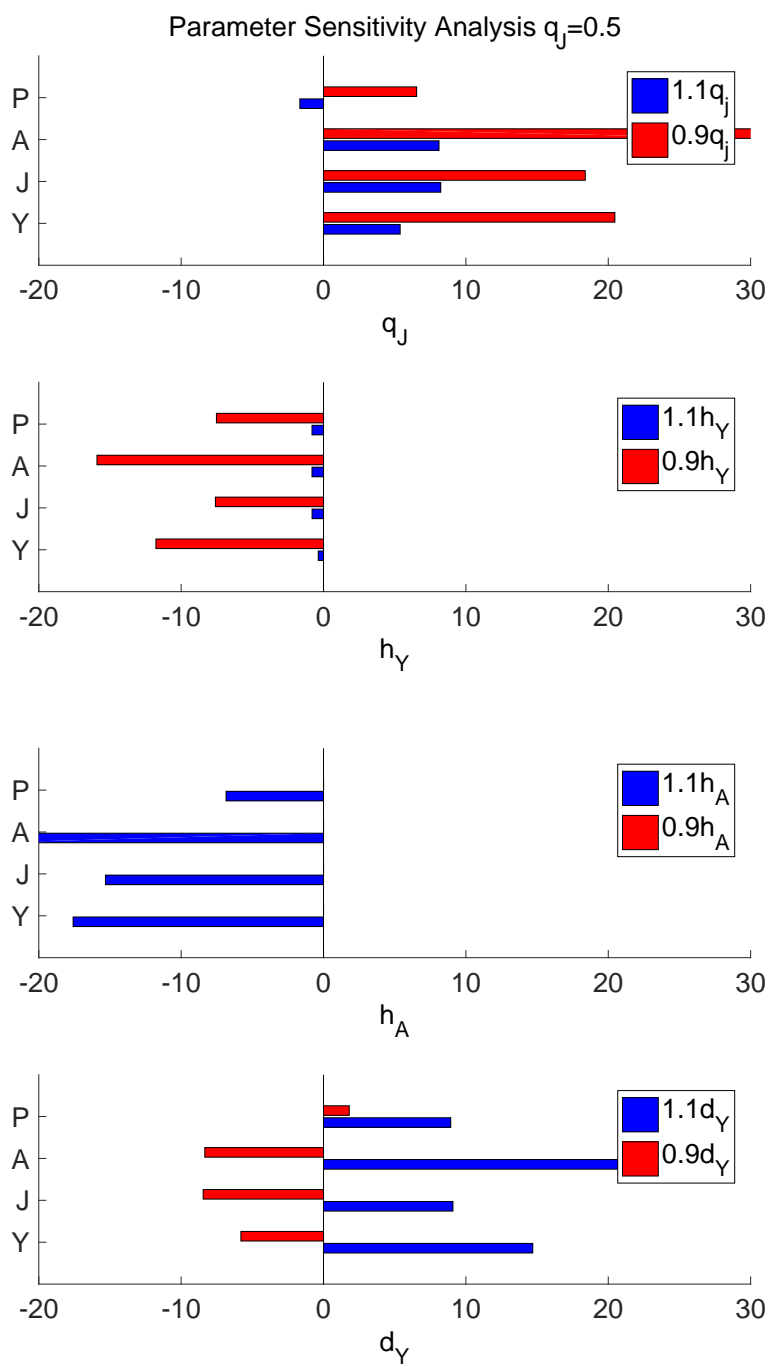

Figure 6: Sensitivity Analysis of $d_{Y}, h_{A}, h_{Y}$ and $q_{J}$ at $q_{J}=0.5$ with $q_{J}$ demonstrating the most significant sensitivity. Blue bars indicate a parameter increase of $10 \%$. Red bars indicate a parameter decrease of $10 \%$. 

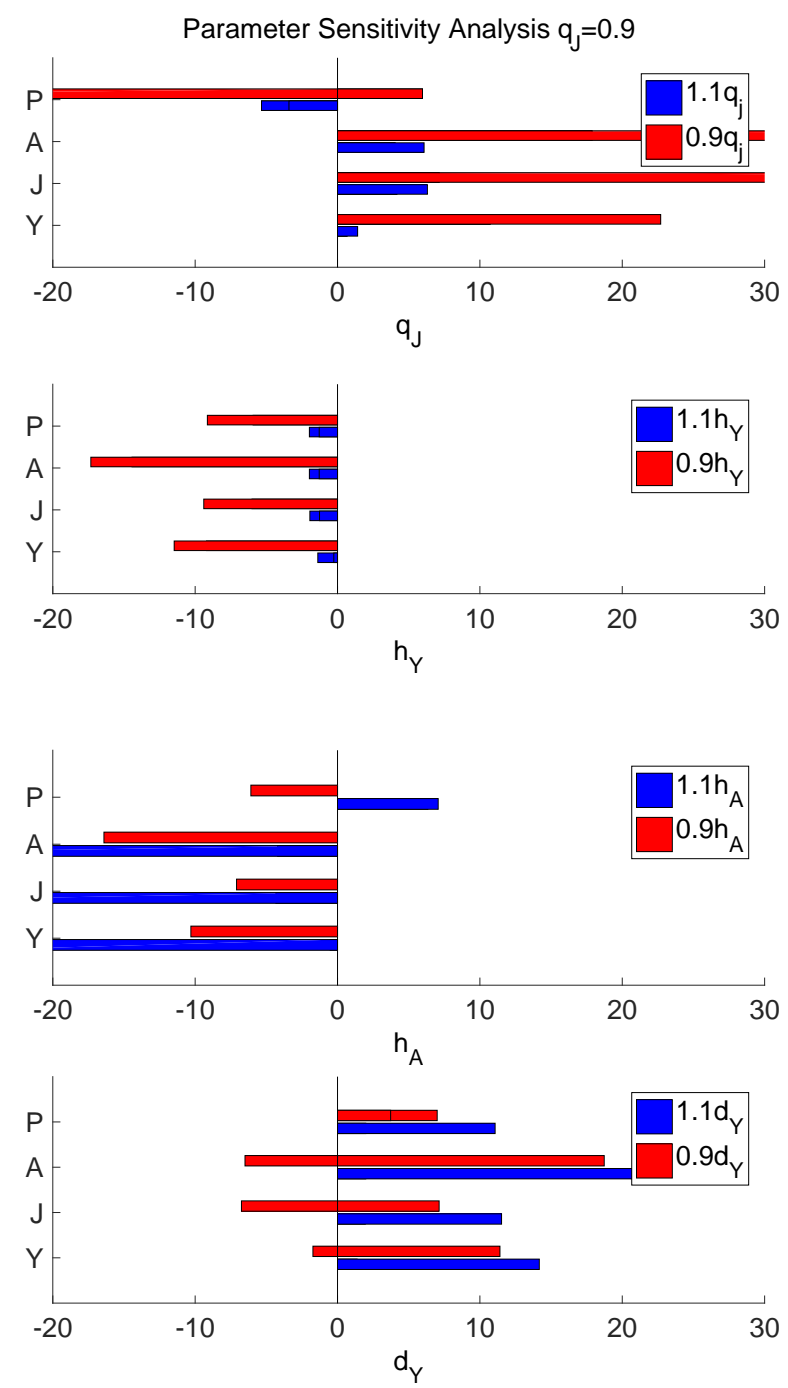

Figure 7: Sensitivity Analysis of $d_{Y}, h_{A}, h_{Y}$ and $q_{J}$ at $q_{J}=0.9$ with $q_{J}$ demonstrating the most significant sensitivity. Blue bars indicate a parameter increase of $10 \%$. Red bars indicate a parameter decrease of $10 \%$. 


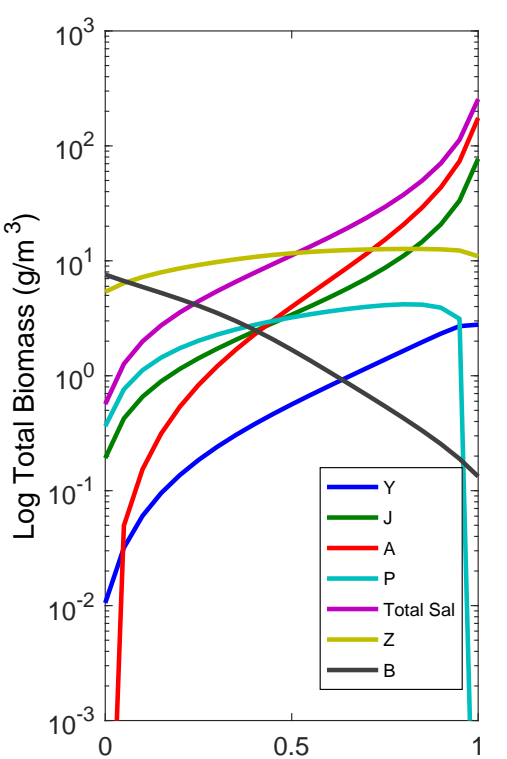

Morphological Choice Parameter Value

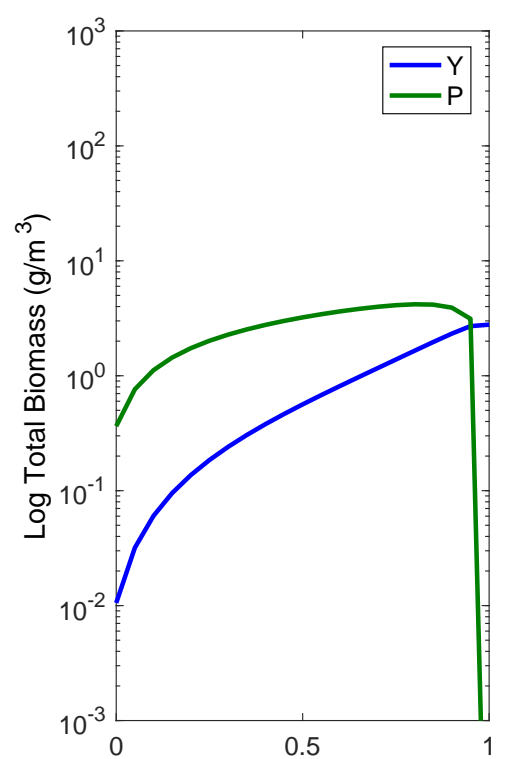

Morphological Choice Parameter Value

Figure 8: Equilibrium pattern in $q_{J}$ for biomass per $m^{3}$ at equilibrium 

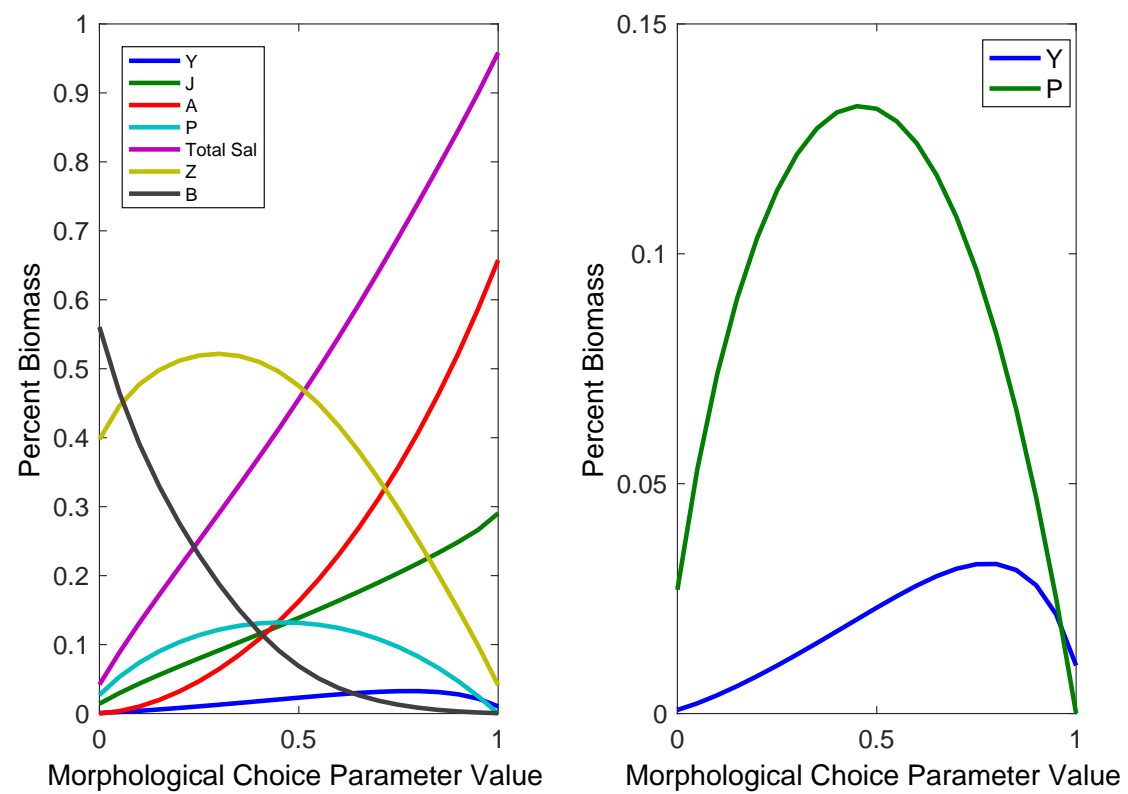

Figure 9: Equilibrium pattern in $q_{J}$ for percent of total biomass at equilibrium 

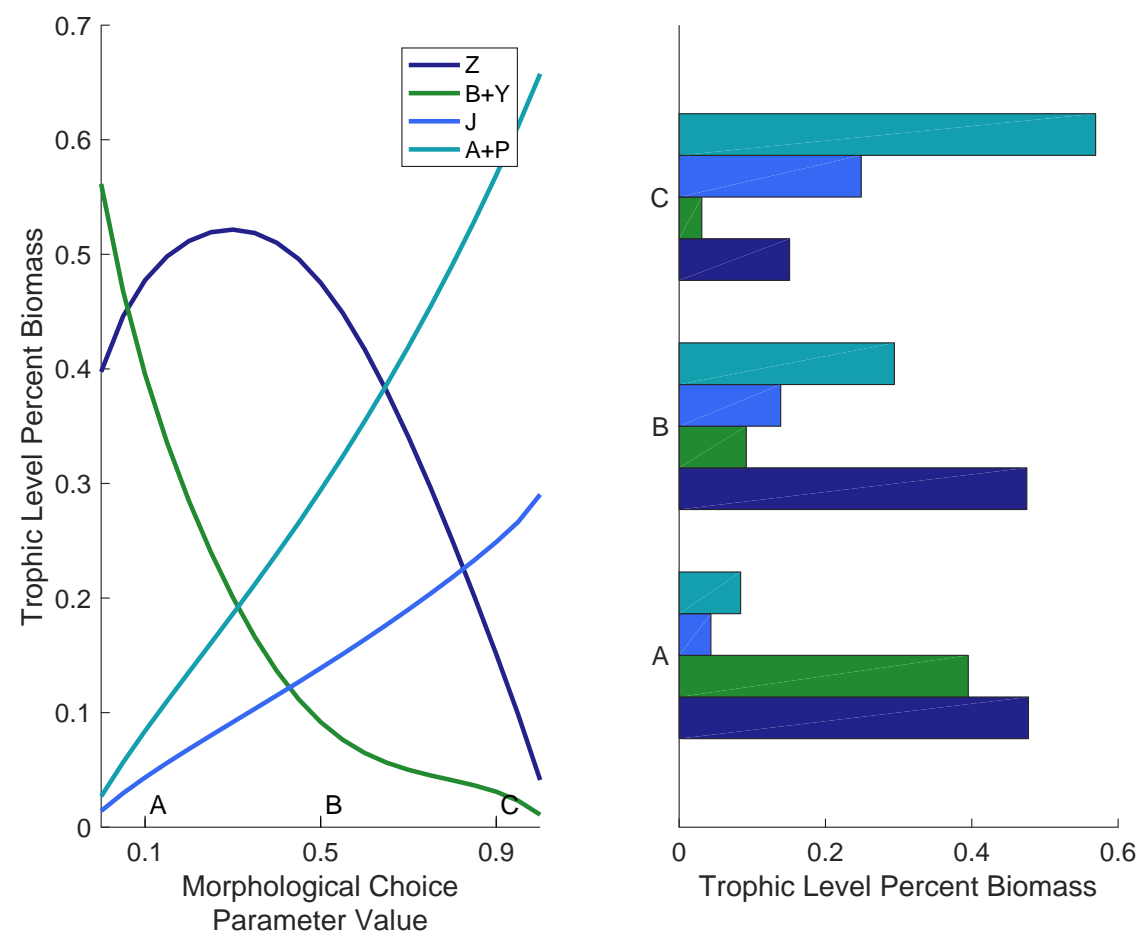

Figure 10: Equilibrium pattern in $q_{J}$ for trophic level biomass, left. Biomass of trophic levels at points $\mathrm{A}, \mathrm{B}, \mathrm{C}$ of equilibrium pattern diagram, right. 